\title{
Logistics Distribution Center Location Optimizatio Model An Example Study
}

\author{
Lingling $\mathrm{Li}^{1{ }^{1}}$ and Jiapeng Zhang ${ }^{1}$ \\ ${ }^{1}$ Management Engineering Department, Zhengzhou University, Zhengzhou, China \\ Corresponding Email: sijiliuzhuan@163.com
}

\begin{abstract}
This paper analyzes and investigates the situation of the today's site of distribution centers .Then taking SF Express for an example ,In the light of minimize the total expense ,the location models for single distribution centers is established with the corresponding resolutions given .After that ,The location of the single distribution center of SF Express ,the starting point of which is Zhengzhou Railway Station ,can be determined with the help of distance-testing function of 360 map to conclude its coordinate and then determine its exact service spot. This paper will test the feasibility of above models by the chosen cases with the application of LINGO software.
\end{abstract}

\section{Introduction}

\subsection{Research background}

In recent years, with the rapid development of China's economy, the computer has become one of the necessary household appliances of every family.

Online shopping has become an indispensable part of people's life. "Internet plus" tide rise rapidly and various industries promote the transformation of enterprises .E-commerce has become an important strategic direction for the future development of enterprises. Distribution center as the most important infrastructure in the process of transport of goods, in the enterprise logistics network planning, the correct location decision is of great importance to reduce the logistics cost.

Distribution center work as a transit point for product transport in the entire logistics network system. It is of great significance to reduce the operating costs of logistics enterprises and improve the service level and efficiency of logistics enterprises. It also has important influence on obtaining enterprise competitive advantage, increasing enterprise efficiency and improving logistics distribution efficiency and customer satisfaction. Scholars at home and abroad for the research of logistics and distribution center are mostly theoretical research. Based on the theoretical research, this paper takes SF Express Company as an example to establish an example model for the cost data collection in a certain distribution area, obtaining the appropriate distribution center location address and applying the theory to the practical application.

\subsection{Domestic and international logistics distribution center location study}

In 1998, Barahona F,etc. established the warehouse location model by using mixed integer programming method which chose the sum of fixed costs, transportation costs, storage costs to be the evaluation criterias [1].

In 1999, Holmberg K studied the location problem on the condition of non-linear transportation cost, and solved the problem by branch-and-bound method [2]; In 2003, Zuo-Jun Max Shen studied the location of distribution center under the condition of joint inventory management, which is a relatively new management model. This model mainly researched the product transport system which the distribution of a supply point deliver a variety of products to multiple customers [3].In 2012, Zhu Rongrong, Hu David considering the characteristics of the cold chain logistics transport items easily degenerate, taking customer satisfaction of logistics services and total logistics costs as the evaluation index, established the cold chain logistics distribution center location model of multi-objective and then solved the research problem with the LINGO software, verifying the availability of this model in location cold chain logistics distribution center [4] ;In 2013, Hu Ziyu, using analytic hierarchy process and Delphi method, studied how to establish a scientific and rational logistics distribution center location model which was based on the theory of economies of scale $[5]$. 
This paper establishes a single distribution center location model with only one distribution center in a specific area, and finally taking SF Express Company as an example, the location of single distribution center is analyzed. Then we input the collected data into LINGO software to solve the model quickly. It proves the usability of the location model in practice, and has certain guiding significance for the construction of distribution centers in county and remote areas of China.

\section{The establishment of a single distribution center location model}

Single distribution center refers to a specific area where only build a distribution center, without regard to the distribution point from the supply point to the distribution center and other issues.

The model is relatively simple. We use the quantitative analysis method to get the optimal distribution center location which take the total cost of the minimum as the principle. First of all, in order to establish the model and make it easier to solve, we make the following assumptions:

(1)The fixed costs of construction and operation of distribution centers are known.

(2)Product distribution costs and distribution quantity is proportional and the cost of the unit product distribution is known.

(3)Distribution center unit product processing costs are known.

(4)The distribution cost is only related to the straight-line distance between the distribution center and the customer

(5)The distribution quantity and location of each customer is known.

(6)Only one product can be delivered and shipped once.

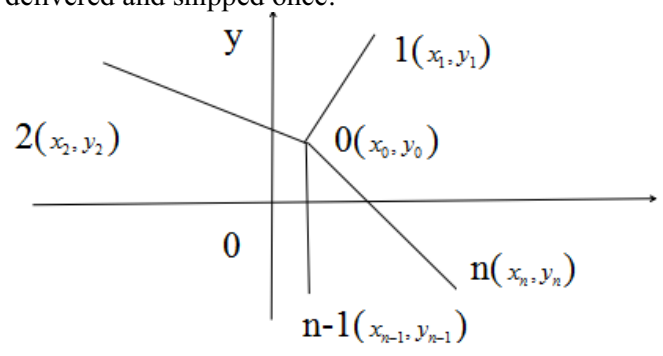

Fig.1. Location model diagram.

A distribution center distributes the goods to multiple distribution points.Our goal is to find the optimal distribution center location where the total cost can be minimized, with known the location of the distribution points.As shown in Figure 1 , there is a distribution point with coordinates $\left(x_{i}, y_{i}\right) \quad(i=1,2,3 \ldots \mathrm{n})$

Assume that the decision variables involved in the model and related parameters are as follows:

The relevant parameters are:

${ }^{w}$ represents distribution center construction, management and other fixed costs; $k$ represents the total distribution quantity of the distribution center (that is the flow of the distribution center), $k_{i}$ represents distribution quantity from the distribution center to the $i$ distribution point; $h_{i}$ represents from the distribution center to the first distribution point of the unit product unit cost of delivery; ${ }^{S}$ represents distribution Center Processing Cost Coefficient; $\theta$ represents the economic benefit index of distribution center,can be taken as $1 / 2$;

The relevant variables : $d_{i}$ represents the straight-line distance between the distribution center and the distribution point $i$.

The total cost from the distribution center to the distribution point is:

$$
\begin{aligned}
& T C=\sum_{i=1}^{n} h_{i} * k_{i} * \sqrt{\left(x_{0}-x_{i}\right)^{2}+\left(y_{0}-y_{i}\right)^{2}}+s * k^{\theta}+w \\
& d_{i}=\sqrt{\left(x_{0}-x_{i}\right)^{2}+\left(y_{0}-y_{i}\right)^{2}} \\
& \text { make }: \frac{\partial T C}{\partial_{x_{0}}}=\sum_{i=1}^{n} \frac{h_{i} k_{i}\left(x_{0}-x_{i}\right)}{d_{i}}=0 \\
& \frac{\partial T C}{\partial_{y_{0}}}=\sum_{i=1}^{n} \frac{h_{i} k_{i}\left(y_{0}-x_{i}\right)}{d_{i}}=0
\end{aligned}
$$

Solution: 


$$
\begin{array}{r}
x_{0}^{*}=\frac{\sum_{i=1}^{n} \frac{h_{i} k_{i} x_{i}}{d_{i}}}{\sum_{i=1}^{n} \frac{h_{i} k_{i}}{d_{i}}} \\
y_{0}^{*}=\frac{\sum_{i=1}^{n} \frac{h_{i} k_{i} y_{i}}{d_{i}}}{\sum_{i=1}^{n} \frac{h_{i} k_{i}}{d_{i}}}
\end{array}
$$

Due to the equations (3-5), (3-6), the right side of the equation ${ }{ }_{i}$ Still contain unknowns ${ } x_{0}, y_{0}$.So a calculation can not be calculated $x_{0}^{*}, y_{0}^{*}$.Generally,given a hypothetical value, and then using the iterative method calculates many times until the minimum total cost $\left(x_{0}, y_{0}\right)$ is found .For the application of gravity method to solve the complex problems, we can input data into the LINGO software to get the answer which greatly reduce the cumbersome degree of calculation.

\section{Example solving - taking SF express location study as an example}

\subsection{Data acquisition}

Using 360 map searches SF Express near the Zhengzhou train station .

From left to right, we using A,B,C,D,E,F represent the delivery points in turn. Taking Zhengzhou Railway Station as the origin, using the 360 map ranging function, Respectively the coordinate of each point are $\mathrm{A}(-2.3,0.9) \quad \mathrm{B}(-2.1,-0.3) \quad \mathrm{C}(-1,-0.8) \quad \mathrm{D}(-0.2,1.1) \quad \mathrm{E}(1.3,-0.5) \quad \mathrm{F}(2,0)$. The coordinate unit is km. We mark the points on the coordinates, As shown in Figure2.

By consulting a staff member of Zhengzhou SF Express distribution center, we learned that the fixed cost of a distribution center for construction and operation is about $w=150000 \mathrm{RMB}$. The distribution cost per $\mathrm{km}$ is approximately $h_{i} k_{i}=2.5$ RMB.Because most of the courier vehicles are self-sufficiency, here we do not consider the distribution center to buy vehicle costs. We have to determine the best location through the coordinates of the distribution center, so we assume that these costs are known and fixed.

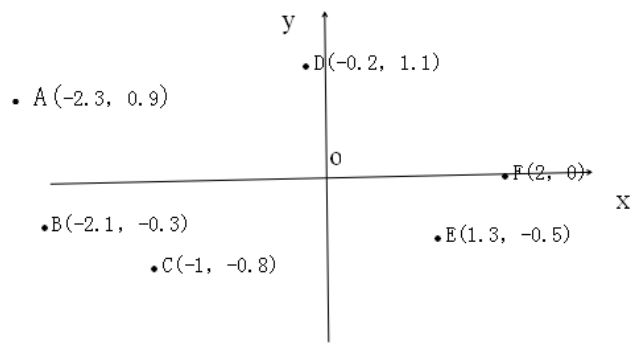

Fig.2. Example coordinate diagram.

\subsection{Based on the study of a single distribution to solve the problem}

Because the model involves so many $\left(x_{i}, y_{i}\right)$, the center of gravity method to solve this problem is more cumbersome.

Therefore, we use LINGO software to solve.LINGO software is from the United States. a company called Lingo System Inc. developed the system whose full name is "Linear Interactive and General Optimizer".It can not only be used to solve nonlinear programming problems quickly, but also can solve some linear and nonlinear equations.the function is very powerful and is used to solving optimization problems.

We do some adjustments for the single delivery center location model. The total monthly cost from the distribution center to the distribution point is:

$$
\min =\sum_{i=1}^{n} 30 * h_{i} * k_{i} * \sqrt{\left(x_{0}-x_{i}\right)^{2}+\left(y_{0}-y_{i}\right)^{2}}+s * k^{\theta}+w
$$




$$
\begin{aligned}
\text { S.T: } d_{i}=\sqrt{\left(x_{0}-x_{i}\right)^{2}+\left(y_{0}-y_{i}\right)^{2}} \\
S * k^{\theta}=15000 \\
h_{i} k_{i}=2.5 \\
w=150000 \\
x_{1}=-2.3 \quad \mathrm{y}_{1}=0.9 \\
x_{2}=-2.1 \quad \mathrm{y}_{2}=-0.3 \\
x_{3}=-1 \quad \mathrm{y}_{3}=-0.8 \\
x_{4}=-0.2 \quad \mathrm{y}_{4}=1.1 \\
x_{5}=1.3 \quad \mathrm{y}_{5}=-0.5 \\
x_{6}=2 \quad \mathrm{y}_{6}=0
\end{aligned}
$$

$x_{i}$ Is an arbitrary value, and $y_{i}$ is an arbitrary value.

The above data input LINGO software, the results are as follows:

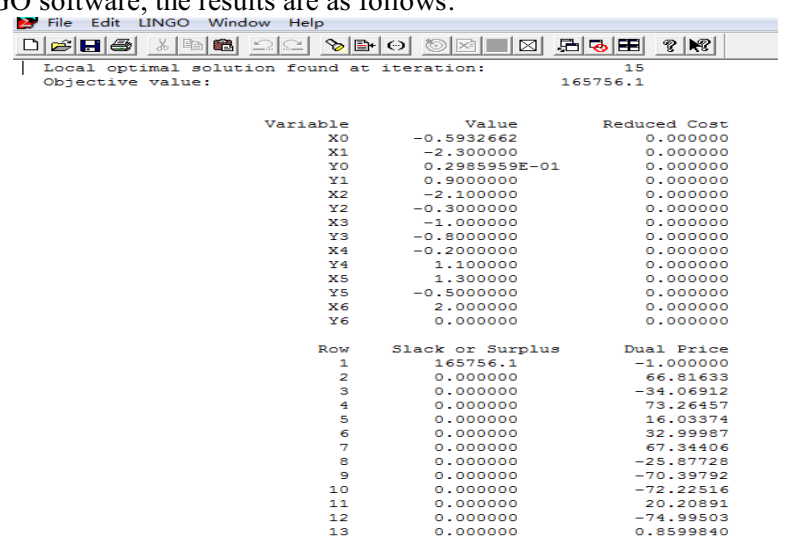

Fig.3. Result output diagram.

$$
x_{0}=-0.5932662 \approx-0.59, y_{0}=0.2985959 \mathrm{E}-01 \approx 0.03 \text {.Distribution center location as shown in Figure } 3 \text {, is shown }
$$
in the middle of Zhengzhou Railway Station about 600 meters .If west.the price is right, you can rent the street facade room location used as SF Express distribution center.

\section{Conclusion}

This paper introduces the location of single distribution center in detail and established single distribution center location model,given the corresponding solution method.

With the help of 360 map,With the help of 360 map, we locate SF Express points near Zhengzhou Railway Station and construct a single distribution center to get the optimal location of distribution center. The innovation of this paper is combined the problem of distribution center location with the concrete examples of SF Express and combine the theory with reality further, strengthening the applicability of the model.It is also of great significance to the establishment of distribution centers in county and remote areas of China.

\section{References}

1. Barahona F.,Jensen D.Plant Location with Minimum Inventory.Mathematical Programming, 1998,83(1):101-111.

2. Holmberg K.Exact Solution Methods for Uncapacitated Location Problem with Convex Transportation Costs.European Journal of Operational Research,1999,114(1):127 -140.

3. Zuo-jun Max shen,Collette Coullard,Mark S.Daskin.Ajoint Location-inventory model.Transportation Science,2003,37(1):40-55. 
4. Zhu Rongrong, Hu Dawei.Multi-objective optimization model of cold chain logistics distribution center selection. Logistics Technology, 2012,32 (01): 108-110.

5. .Hu Ziyu.Study on the Location of Logistics Distribution Center under Scale Economy .Commercial Times, 2013,32 (31): 29-30. 